\title{
Cyclotron orbit knot and tunable-field quantum Hall effect
}

\author{
Yi Zhang $\oplus^{*}$ \\ International Center for Quantum Materials, Peking University, Beijing 100871, China; \\ School of Physics, Peking University, Beijing 100871, China; \\ Department of Physics, Cornell University, Ithaca, New York 14853, USA; \\ and Kavli Institute for Theoretical Physics, University of California, Santa Barbara, California 93106, USA
}

(Received 17 May 2019; published 16 September 2019)

\begin{abstract}
From a semiclassical perspective, the Bohr-Sommerfeld quantization of the closed cyclotron orbits for charged particles such as electrons in an external magnetic field gives rise to discrete Landau levels and a series of fascinating quantum Hall phenomena. Here, we consider topologically nontrivial physics from a distinct origin, where the cyclotron orbits take nontrivial knotting structures such as a trefoil knot. We present a scenario of a Weyl semimetal with a slab geometry, where the Fermi arcs on the opposing surfaces can cross without interfering with each other and form a knot together with the bulk Weyl nodes, and in an external magnetic field, the resulting chiral Landau levels. We provide a microscopic lattice model to realize a cyclotron orbit with an unconventional geometry of a trefoil knot and study the corresponding quantum oscillations. Interestingly, unlike the conventional ring-shaped cyclotron orbit, a trefoil knot is self-threading, allowing the magnetic field line along the cyclotron orbit to contribute to the overall Berry phase and therefore altering the external magnetic field for each quantization level. The cyclotron orbit knot offers an arena of the nontrivial knot theory in three spatial dimensions and its subsequent physical consequences.
\end{abstract}

DOI: 10.1103/PhysRevResearch.1.022005

Integer quantum Hall effect [1] and the subsequently discovered zoo of miscellaneous topological phases [2-10] represent an active research area in condensed matter physics. The physics idea, however, may trace back further to the insightful semiclassical quantization of the cyclotron orbits $[11,12]$. Following the semiclassical equations of motion, charged particles such as electrons cycle in the plane normal to the magnetic field around the constant energy contours, which then become quantized according to the Bohr-Sommerfeld quantization condition. In two dimensions, all electron degrees of freedom are quenched into these cyclotron orbits with discrete and degenerate energy values - the Landau levels-in a magnetic field. The spacing and degeneracy of the Landau levels are proportional to the magnetic field strength, giving rise to the oscillations in material electronic properties as a function of the applied magnetic field such as de Haas-van Alphen effect, Shubnikov-de Haas effect, and other fascinating facets of the quantum Hall effect.

A relatively recent series in topological materials is the topological Weyl and Dirac semimetals in three dimensions $[9,10]$. Around the Weyl nodes (selected points in their Brillouin zone), the low-energy electronic excitations disperse linearly, resembling the Weyl fermions in models for highenergy physics. Also, the surface electronic states consist of

\footnotetext{
*frankzhangyi@gmail.com

Published by the American Physical Society under the terms of the Creative Commons Attribution 4.0 International license. Further distribution of this work must maintain attribution to the author(s) and the published article's title, journal citation, and DOI.
}

exotic open Fermi arcs $[9,10]$. In the presence of a magnetic field, the Weyl fermions become quantized as the chiral Landau levels that disperse along or against the magnetic field, depending on the Weyl fermions' chirality, and they exhibit the chiral anomaly phenomenon [13]. These chiral Landau levels, together with the Fermi arcs on the top and bottom surfaces in a slab geometry, assemble a cyclotron orbit in the Dirac and Weyl semimetals, dubbed the Weyl orbit [14,15]; see Fig. 1(a) for illustration. The Weyl orbit extends in and promotes the Landau quantization to three spatial dimensions, and the corresponding quantum oscillations [16] and quantum Hall transports [17-19] have been established experimentally.

The search for topological phenomena in condensed matter physics $[8,20]$ has received much inspiration from the mathematical studies on topology such as knot theory, which investigates the nonequivalent classes of closed loops and the corresponding invariants, e.g., the Jones polynomial, in higher dimensional spaces. Indeed, a series of two-dimensional topological orders are characterized by topological quantum field theory on nontrivial quasiparticle world-line knots in $(2+1)$ dimensional space-time [20]. Also, the topologically distinct fermionic excitations in the form of connected links and knots in momentum space are discovered in nodal link [21-25] and nodal knot $[24,26]$ metals. In this work, we present our discovery of a topology origin of quantum materials and phenomena, where the cyclotron orbit employs a nontrivial knot topology, such as a trefoil knot [Fig. 1(c)], in three spatial dimensions. Commonly, a cyclotron orbit is self-evading, since the couplings between nearby Fermi surfaces generally widen the gap and move them further apart, especially in a magnetic field, making crossings-a key ingredient of knots-difficult to realize. The Weyl orbits in the Dirac and Weyl semimetal 
(a)

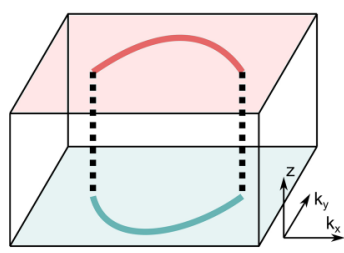

(c)

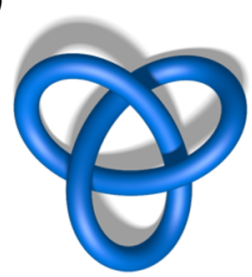

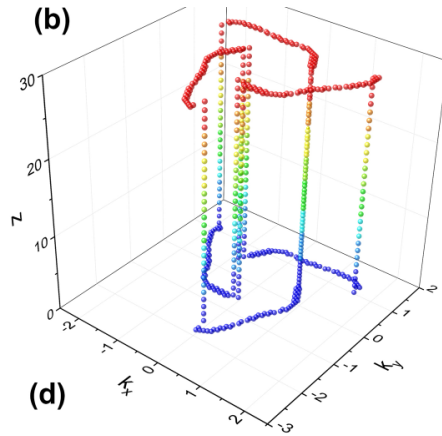

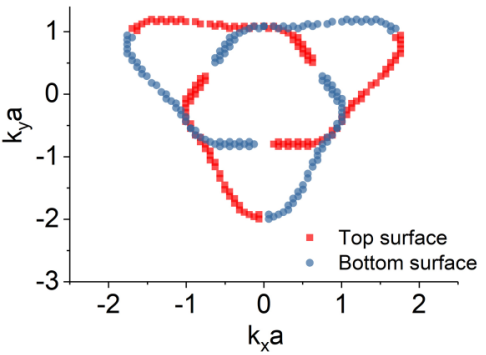

FIG. 1. (a) The Weyl orbit consists of the Fermi arcs on the top (red) and bottom (blue) surfaces and the chiral Landau levels from the Weyl nodes in the bulk (black dashed lines). This Weyl orbit is still topologically equivalent to a conventional ring-shaped cyclotron orbit. (b) The Fermi arcs of the Weyl semimetal model in Eq. (1) on the top and bottom surfaces of a slab and the bulk tunneling at the six Weyl nodes assemble a cyclotron orbit with a trefoil-knot geometry. For better visibility, the points show the values of $\left(k_{x}, k_{y}, z\right)$ for low-energy states within an energy window $E \in[-0.1,0.1]$ around the Weyl nodes. In the presence of a magnetic field along $\hat{z}$, the shape of the cyclotron orbit in three spatial dimensions is related by a 90-deg rotation in the $x y$ plane. (c) A trefoil knot is a closed loop with three crossings and topologically distinctive from a ring-shaped loop. (d) The projection of panel (b) onto the $k_{x}, k_{y}$ plane demonstrates the three crossings similar to those in panel (c). Thanks to the spatial separation between the surfaces, the Fermi arcs manage to cross without interfering with each other.

slabs offer a solution to this difficulty, as the Fermi arcs on the top and bottom surfaces are spatially separated and can form crossings without interfering with each other. We provide a microscopic lattice model example where we realize a Weyl orbit with a trefoil knot geometry; see Figs. 1(b) and 1(d) [27]. In three spatial dimensions, such a cyclotron orbit knot is not adiabatically connected to a conventional ring-shaped cyclotron orbit, including the conventional Weyl orbit, shown in Fig. 1(a).

The nontrivial topology of the cyclotron orbit knot also has profound physical consequences. For instance, unlike a ringshaped loop, a knot is self-threading. Therefore, a magnetic field line along a cyclotron orbit knot contributes nontrivially to the overall Berry phase around the orbit. Tuning this flux allows the contribution from other sources such as the magnetic field to differ in order to reach a specific Landau quantization. Using our microscopic lattice model, we study the behavior of the quantum oscillations associated with the cyclotron orbit knot. In particular, we introduce a perturbation that creates an effective magnetic field that aligns with the

electronic velocity and study its impact on the subsequent quantum Hall effect.

Without loss of generality, we consider an electronic tightbinding model on a three-dimensional hexagonal lattice for concreteness:

$$
\begin{aligned}
H= & \sum_{\langle i j\rangle, z, s} t(-1)^{z} c_{j z s}^{\dagger} c_{i z s}+\sum_{\langle\langle i k\rangle\rangle, z, s} t_{i k}^{\prime}(-1)^{z} \sigma_{s s}^{z} c_{k z s}^{\dagger} c_{i z s} \\
& +\sum_{i,\left\langle z z^{\prime}\right\rangle, s} t_{z} \sigma_{s s}^{z} c_{i z^{\prime} s}^{\dagger} c_{i z s}+\sum_{\langle i k\rangle,\left\langle z z^{\prime}\right\rangle, s} t_{i z k z^{\prime}}^{\prime \prime}(-1)^{z} c_{k z^{\prime} s}^{\dagger} c_{i z s} \\
& +\sum_{i, z^{\prime}=z+1, s, s^{\prime}} i \Delta \sigma_{s s^{\prime}}^{x}\left(c_{i z^{\prime} s^{\prime}}^{\dagger} c_{i z s}-c_{i z s}^{\dagger} c_{i z^{\prime} s^{\prime}}\right) \\
& -\sum_{i, z, s} \mu_{s}(-1)^{z} c_{i z s}^{\dagger} c_{i z s}
\end{aligned}
$$

where $i, j, k$ are the coordinates in the $x y$ plane and $\sigma$ are the Pauli matrices defined on the two pseudospins $s, s^{\prime}=\uparrow, \downarrow$. The first two terms are hoppings in the $x y$ plane between the nearest neighbors and the next nearest neighbors, $t_{i k}^{\prime}= \pm i t^{\prime}$, and the next two terms are near-neighbor hoppings between the nearest layers, $t_{i z k z^{\prime}}^{\prime \prime}= \pm i t_{z}^{\prime}$, where the $\pm i=\exp \left(3 i \phi_{i k}\right)$ phases depend on the azimuthal angle $\phi_{i k}$ from $i$ to $k$ [28]. The last two terms are a coupling between the two pseudospins $s, s^{\prime}=\uparrow, \downarrow$ and a chemical potential. In the rest of the paper, we set $t=-1.0, t^{\prime}=-0.5, t_{z}=-1.0, t_{z}^{\prime}=-0.5, \Delta=0.4$, $\mu_{\uparrow}=-5.1$, and $\mu_{\downarrow}=-3.5$ unless noted otherwise. All lattice constants are set to 1 .

First of all, this model system is a Weyl semimetal. To see this, we transform Eq. (1) into the momentum space $H=\sum_{\mathbf{k}} H_{\mathbf{k}}$, with

$$
\begin{aligned}
H_{\mathbf{k}}= & \tau^{z}\left[\epsilon_{0}\left(k_{\perp}\right)+\sigma^{z} \epsilon_{z}\left(k_{\perp}\right)\right]+\tau^{x} \sigma^{z} 2 t_{z} \cos k_{z} \\
& +\tau^{y} 4 t_{z}^{\prime} \sin k_{z}\left(\sin k_{1}+\sin k_{2}+\sin k_{3}\right)-\tau^{x} \sigma^{x} 2 \Delta \sin k_{z}
\end{aligned}
$$

where $\tau$ are the Pauli matrices on the even-odd layers. $k_{z} \in$ $[0, \pi)$ is the momentum along the $\hat{z}$ direction, and $k_{1}=k_{x}$, $k_{2}=\left(-k_{x}+\sqrt{3} k_{y}\right) / 2, k_{3}=\left(-k_{x}-\sqrt{3} k_{y}\right) / 2$ are momenta in the $x y$ plane. $\epsilon_{0}\left(k_{\perp}\right)+\sigma^{z} \epsilon_{z}\left(k_{\perp}\right)$ are the Fourier transform of the $t, t^{\prime}$, and $\mu_{s}$ terms. There are six Weyl nodes on the $k_{z}=$ $\pi / 2$ plane at $\left(k_{x}, k_{y}\right)=(0,-1.96)$ with $\sigma_{z} \simeq \uparrow$ and $\left(k_{x}, k_{y}\right)=$ $(0,-0.834)$ with $\sigma_{z} \simeq \downarrow$ and their counterparts after the $C_{3}$ rotations [29]. The band gap closes at these Weyl nodes, and the low-energy dispersion around them is linear. Interestingly, for a model system with a finite thickness $L_{z}$ along the $\hat{z}$ direction, the surface Fermi arcs consist of three Fermi arcs on the top surface and three on the bottom surface. The $\left(k_{x}, k_{y}, z\right)$ location of the Fermi arcs and the bulk states at the Weyl nodes are shown in Fig. 1(b) for $L_{z}=29$, thick enough to separate the Fermi arc states on the opposing surfaces. Therefore, the constant energy contour employs a trefoil knot [Fig. 1(c)], which engages all six Weyl nodes and six Fermi arcs at once.

In the presence of an applied magnetic field along $\hat{z}$, the electrons cycle around the cyclotron orbit, whose shape in three spatial dimensions is related by a 90-deg rotation in the $x y$ plane. Therefore, we have established a cyclotron orbit with a trefoil knot geometry that is topologically distinctive and not adiabatically connected with the conventional ringshaped cyclotron orbits. We note the important role the Weyl 


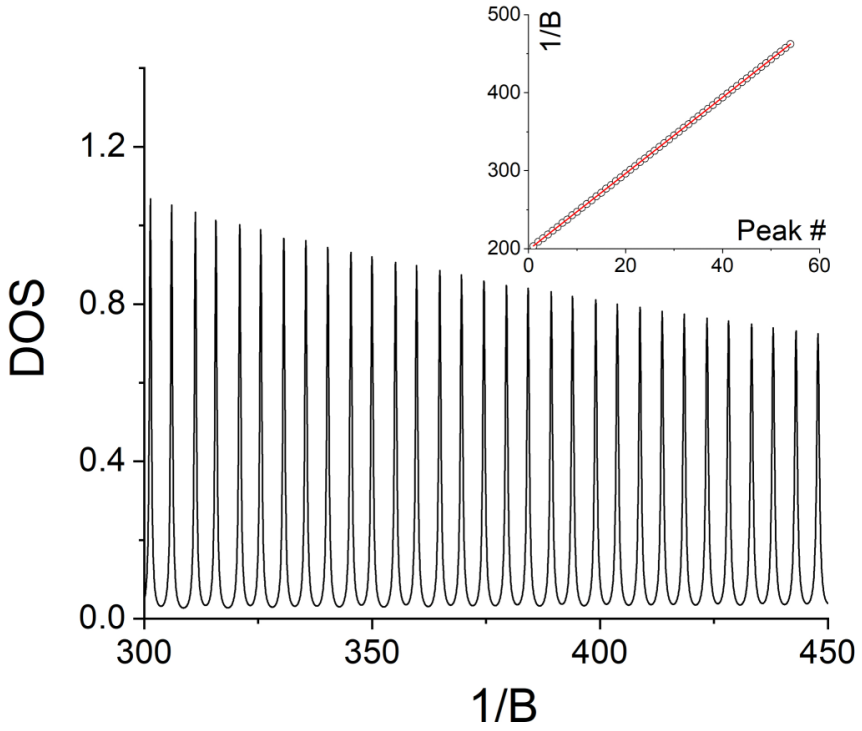

FIG. 2. The DOS vs the inverse magnetic field $1 / B$ shows clear quantum oscillations with a single period. The Hamiltonian in Eq. (1) with a magnetic vector potential $\vec{A}=(-B y, 0)$ is calculated using the recursive Green's function method. We focus on the chemical potential at the Weyl nodes and consider a slab with thickness $L_{z}=29$. A small imaginary part $\delta=0.001$ is added to the energy to account for a finite level width. Inset: a linear fit to the peak position within the $200<1 / B<500$ range reveals a quantum oscillation period of $\Delta(1 / B) \simeq 4.87$.

orbit physics plays in attaining cyclotron orbit knots: The top and bottom Fermi arcs are spatially separated and can safely traverse each other without being gapped out even in the presence of a variable magnetic field. Together with the bulk chiral Landau levels that descend from the Weyl nodes and weave the two surfaces back together, we can form crossings that are the cornerstones for knots and links. The construction of trefoil-knot-shaped cyclotron orbit can be straightforwardly generalized to cyclotron orbits with more complex knots and links.

Next, we study the Landau quantization of the cyclotron orbit knot and consider the Hamiltonian in Eq. (1) with a slab thickness of $L_{z}=29$ in the presence of a magnetic vector potential $\vec{A}=(-B y, 0)$. Physical quantities such as the density of states (DOS) at the energy of the Weyl nodes can be obtained using the recursive Green's function method for sufficiently large system sizes. The results on the DOS versus the inverse magnetic field are summarized in Fig. 2. We observe a single quantum oscillation period of $\Delta(1 / B)=$ $1 / B_{n+1}-1 / B_{n} \simeq 4.87$. Since the bulk chiral Landau levels are parallel to the magnetic field, the quantum oscillations of a Weyl orbit is determined by the area $S_{k}$ of the combined Fermi arcs from the top and bottom surfaces [15]. Interestingly, after projection and combination of the Fermi arcs [Fig. 1(d)], the area within the inner contour is $S_{k 2} \sim 4.78 \% \times S_{B Z}$ of the surface Brillouin zone area $S_{B Z}$, and the area within the outer contour (including $S_{k 2}$ ) is $S_{k 1} \sim 15.2 \% \times S_{B Z}$. That $\Delta(1 / B) \simeq\left(S_{k 1}+S_{k 2}\right)^{-1}=5.0$ indicates the magnetic flux enclosed in the inner contour contributes to the overall Berry phase twice. Indeed, straightforward counting suggests that (a)

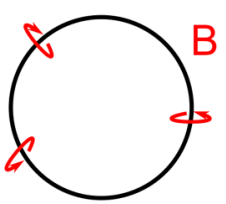

(b)

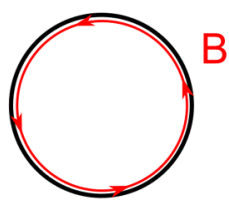

(c)

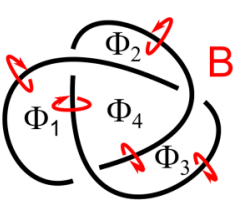

(d)

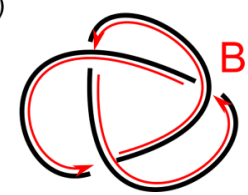

FIG. 3. The magnetic field line $\mathbf{B}$ (red arrows) for ring-shaped and trefoil-knot-shaped cyclotron orbits. (a) A magnetic field line through the ring contributes a Berry phase. (b) The magnetic field line along the ring-shaped cyclotron orbit has no Berry phase contribution. (c) The magnetic field line through the trefoil knot contributes a Berry phase. The contributions from the fluxes in different regions $\Phi_{\text {total }}=\sum_{i=1}^{4} \alpha_{i} \Phi_{i}$ obey $\alpha_{1}=\alpha_{2}=\alpha_{3}=\alpha_{4} / 2$. (d) Since a trefoil knot winds around itself, the magnetic field line along the cyclotron orbit knot now contributes a nonzero Berry phase.

the cyclotron orbit encloses the inner region twice upon each cycle. More rigorously, the contributions to the overall Berry phase $\Phi_{\text {total }}=\sum_{i=1}^{4} \alpha_{i} \Phi_{i}$ obeys $\alpha_{1}=\alpha_{2}=\alpha_{3}=\alpha_{4} / 2$, since the adiabatic change as illustrated in Fig. 3(c) of a magnetic field loop with flux $\Phi$ indicates the equivalence $\alpha_{1} \Phi=$ $\alpha_{2} \Phi=\alpha_{3} \Phi=\left(\alpha_{4}-\alpha_{1}\right) \Phi=\left(\alpha_{4}-\alpha_{3}\right) \Phi$. On that account, our numerical results on quantum oscillations are fully consistent with the trefoil knot geometry of the cyclotron orbit.

Then, we discuss an interesting physical consequence of the nontrivial knot geometry of a cyclotron orbit: the tunablefield quantum Hall effect. Conventionally, the magnetic field line along the ring-shaped cyclotron orbit does not contribute to the overall Berry phase [Fig. 3(b)]. In contrast, a trefoil knot is self-threading, and thus the magnetic field line along the cyclotron orbit knot contributes nontrivially to the overall Berry phase; see Fig. 3(d). By controlling the contribution from such flux, we can modify the Landau quantization condition for other sources of the Berry phase, such as the applied magnetic field. The conventional spin-orbit interaction introduces a $\vec{v}$ dependent effective magnetic field that couples to the electron spin; here, we need to introduce a $\vec{v}$-dependent effective magnetic field $\vec{B}_{\text {eff }}$ that couples to the electron orbital angular momentum. One of the electron semiclassical equations of motion $d \vec{r} / d t=\vec{v}(\vec{k})$ indicates that the cyclotron orbit is parallel to electron velocity at every instance and guarantees an effective magnetic field $\vec{B}_{\text {eff }} \| \vec{v}$, long overlooked due to the presence of only $\vec{B} \times \vec{v}$ term in the equations of motion $[11,12,30]$, is along the cyclotron orbit irrespective of the shape details and contributes to the tunable-field quantum Hall effect.

In the case of a knotting Weyl orbit, since the Fermi arcs locally responsible for the crossings are on the surfaces, we limit our attention to the effective magnetic fields in the $x y$ plane. For instance, along the $\hat{y}$ direction, we may introduce a $\vec{v}$-dependent effective magnetic field with a perturbation $H^{\prime} \propto v_{y}(\vec{k}+\eta z \hat{x})-v_{y}(\vec{k}-\eta z \hat{x}) \simeq 2 i \eta z\left[x, v_{y}\right]$ for small $\eta$, where $v_{y}=i[y, H]$ is the electron velocity in the $\hat{y}$ 
direction. However, it leads to a $\sin \left(k_{3}\right)-\sin \left(k_{2}\right)$ azimuthal angle dependence and vanishes after taking into account the $-\frac{1}{2} \hat{y} \pm \frac{\sqrt{3}}{2} \hat{x}$ directions. Therefore, we introduce an additional factor of $\cos \left(k_{1}\right)$, which is $\approx 1$ and a good approximation near the Weyl nodes and the crossing point where $v_{y}$ is the most important. After similar treatment to the $-\frac{1}{2} \hat{y} \pm \frac{\sqrt{3}}{2} \hat{x}$ directions, we obtain in total

$$
H^{\prime}=\sum_{\langle\langle i k\rangle\rangle, z^{\prime}=z+1, s} t_{z}^{\prime} \exp \left(3 i \phi_{i k}\right) c_{k z^{\prime} s}^{\dagger} c_{i z s} \times \eta z(-1)^{z}+\text { H.c. }
$$

For slowly varying $\eta z, H^{\prime}=\sum_{\mathbf{k}} H_{\mathbf{k}}^{\prime}$ takes a momentum-space form,

$$
\begin{aligned}
H_{\mathbf{k}}^{\prime} \simeq & \tau^{y} 4 t_{z}^{\prime} \eta z \sin k_{z}\left[\sin \left(k_{2}-k_{1}\right)+\sin \left(k_{3}-k_{2}\right)\right. \\
& \left.+\sin \left(k_{1}-k_{3}\right)\right],
\end{aligned}
$$

that only has $\tau^{y}$ components and thus does not interfere with the quantum oscillation period, which is determined by the combined surface Fermi arcs [15] and the $\tau^{z}$ terms in the original $H_{\mathbf{k}}$ in Eq. (3). However, $H^{\prime}$ does have an interesting impact on the model physics.

As we include the perturbation $H^{\prime}$ into the original Hamiltonian $H$ in Eq. (1), the magnetic field $B$ corresponding to each Landau level changes. We track the shift of the DOS quantum oscillation peak $\Delta\left(1 / B_{\text {peak }}\right)=1 / B_{\text {peak }}(\eta)-1 / B_{\text {peak }}(\eta=0)$ as a function of $\eta$, and the results are summarized in Fig. 4. The ratio $\Delta\left(1 / B_{\text {peak }}\right) /\left(1 / B_{n+1}-1 / B_{n}\right)$ measures the Berry phase contributed by the perturbation in unit of the magnetic flux quantum. On the other hand, the period of the quantum oscillations remains unchanged; see Fig. 4 inset. Linear fits to 40 peak positions within the $200<1 / B<400$ range indicate an identical period of $\Delta(1 / B) \simeq 4.87$ for $\eta=$ $0, \pm 0.01, \pm 0.02$. Therefore, the inclusion of $H^{\prime}$ induces an extra yet constant phase to the cyclotron orbit knot.

In fact, the tunable-field quantum Hall effect is not fully unheard of - the overall Berry phase of the Weyl orbit receives a contribution from the bulk chiral Landau levels that depends on the Fermi energy, the tilting direction of the applied magnetic field, and the thickness of the system $[15,19]$. The extra Berry phase in this work, however, is consequential to the nontrivial knotting topology of the cyclotron orbit and comes from a completely different origin: With or without the $H^{\prime}$ perturbations, our model systems are still $C_{3}$ rotation symmetric, the Fermi energy is at the Weyl nodes, and the magnetic field is along $\hat{z}$ without tilting. Indeed, this contribution is unique to the cyclotron orbit knot model. In contrast, such an in-plane effective magnetic field incurs no orbital effect for conventional cyclotron orbits in two-dimensional electron systems. Further, we repeat the calculations for the $\sigma^{z}=\uparrow$ sector of the original Hamiltonian $H$ in Eq. (1), which describes a conventional Weyl semimetal with a topologicallyring-shaped Weyl orbit. Its Weyl orbit also consists of three pairs of chiral Landau levels and three pieces of Fermi arcs on each of the top and bottom surfaces. As we change the amplitude $\eta$ of the perturbation, given by the $\sigma^{z}=\uparrow$ sector of $H^{\prime}$ in Eq. (3), the DOS peaks in the quantum oscillations hardly shift; see the black diamond symbols in Fig. 4. The

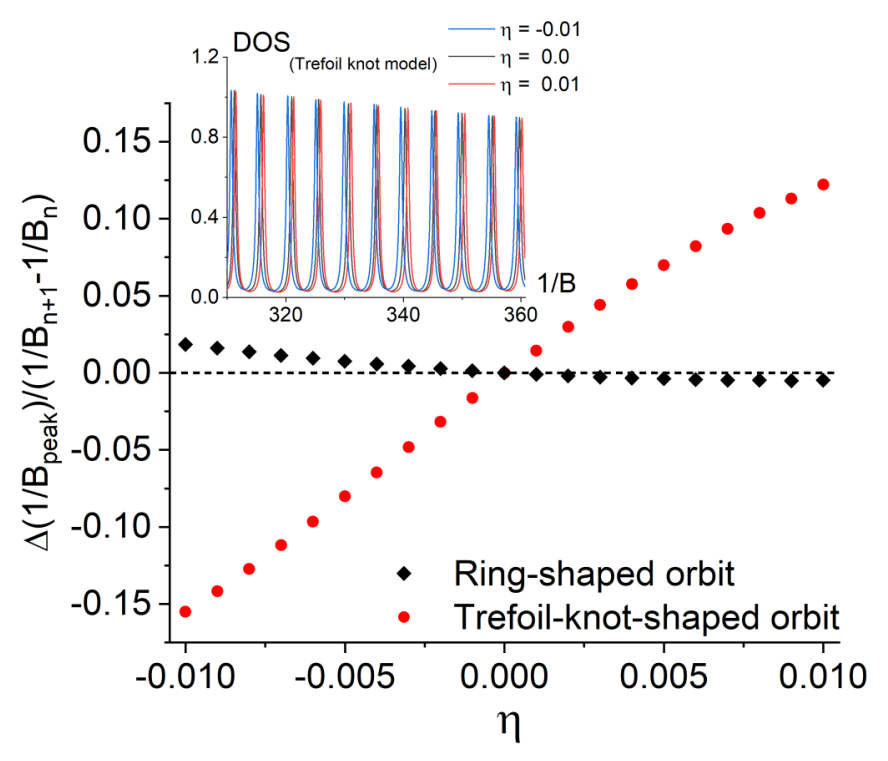

FIG. 4. The shifts of the DOS quantum oscillation peak positions $1 / B_{\text {peak }}$ vs the amplitude $\eta$ of the applied perturbation in Eq. (3). We track the DOS peak at $1 / B \sim 227.78$ at $\eta=0$ for the cyclotron orbit knot model and the DOS peak at $1 / B \sim 223.61$ at $\eta=0$ for the conventional Weyl orbit model as the $\sigma^{z}=\uparrow$ sector of $H+H^{\prime}$ in Eqs. (1) and (3). The perturbation creates an effective magnetic flux that contributes to the cyclotron orbit knots and changes the Landau quantization condition for the magnetic field; see the red dots. In comparison, the $1 / B$ quantization condition, shown as the black diamonds, hardly changes for the conventional ring-shaped Weyl orbit. Inset: the DOS peaks for the cyclotron orbit knot models displace with $\eta$ yet retain an identical quantum oscillation period of $\Delta(1 / B) \simeq 4.87$ for $\eta=0, \pm 0.01$.

small deviations from ideal theory expectation may be due to the approximation in $H^{\prime}$.

In conclusion, we have shown a topological prospect where the cyclotron orbit takes a nontrivial knot geometry in three spatial dimensions and proposed the Weyl semimetal slab as an example of realization. We have also provided a microscopic lattice model, investigated its Landau quantization, and demonstrated that the unusual knotting topology of the cyclotron orbit allows the continuous tuning of the magnetic field condition for quantum Hall effect. We note that the Weyl orbits depend on the surface states, and thus the cyclotron orbit knot can be realized on existing Weyl semimetals, such as the RhSi with large Fermi arcs [31] via proper surface design [28].

Since the knots are topologically protected and invariant in three spatial dimensions, a cyclotron orbit knot is not adiabatically connected with the conventional ring-shaped cyclotron orbits [28] and is allowed to have characteristic topological properties. In topological quantum field theory, the nontrivial anyonic phases of quasiparticle braiding and statistics in two spatial dimensions are consequential to the nontrivial Jones polynomial of the knots between the quasiparticle world lines in $(2+1)$-dimensional space time [20]. The consequences of cyclotron orbit knots in three spatial dimensions on characteristic properties, such as intrinsic Berry phase contributions [30], the scenarios beyond $U(1)$ gauge field, etc., and their 
connections to the knot invariants, are interesting further directions for future exploration.

Y.Z. acknowledges support from the start-up grant at International Center for Quantum Materials, Peking
University, the Bethe fellowship at Cornell University, and the National Science Foundation under Grant No. NSF PHY-1748958. The computation was supported by High-Performance Computing Platform of Peking University.
[1] K. V. Klitzing, G. Dorda, and M. Pepper, Phys. Rev. Lett. 45, 494 (1980).

[2] D. C. Tsui, H. L. Stormer, and A. C. Gossard, Phys. Rev. Lett. 48, 1559 (1982).

[3] R. B. Laughlin, Phys. Rev. Lett. 50, 1395 (1983).

[4] F. D. M. Haldane, Phys. Rev. Lett. 61, 2015 (1988).

[5] C. L. Kane and E. J. Mele, Phys. Rev. Lett. 95, 146802 (2005).

[6] L. Fu, C. L. Kane, and E. J. Mele, Phys. Rev. Lett. 98, 106803 (2007).

[7] M. Z. Hasan and C. L. Kane, Rev. Mod. Phys. 82, 3045 (2010).

[8] X. Chen, Z.-C. Gu, Z.-X. Liu, and X.-G. Wen, Science 338, 1604 (2012).

[9] X. Wan, A. M. Turner, A. Vishwanath, and S. Y. Savrasov, Phys. Rev. B 83, 205101 (2011).

[10] S.-Y. Xu, I. Belopolski, N. Alidoust, M. Neupane, G. Bian, C. Zhang, R. Sankar, G. Chang, Z. Yuan, C.-C. Lee, S.-M. Huang, H. Zheng, J. Ma, D. S. Sanchez, B. Wang, A. Bansil, F. Chou, P. P. Shibayev, H. Lin, S. Jia, and M. Z. Hasan, Science 349, 613 (2015).

[11] L. Onsager, London, Edinburgh, Dublin Philos. Mag. J. Sci. 43, 1006 (1952).

[12] I. Lifshitz and A. Kosevich, J. Exp. Theor. Phys. 2, 636 (1956).

[13] H. Nielsen and M. Ninomiya, Phys. Lett. B 130, 389 (1983).

[14] A. C. Potter, I. Kimchi, and A. Vishwanath, Nat. Commun. 5, 5161 (2014).

[15] Y. Zhang, D. Bulmash, P. Hosur, A. C. Potter, and A. Vishwanath, Sci. Rep. 6, 23741 (2016).

[16] P. J. W. Moll, N. L. Nair, T. Helm, A. C. Potter, I. Kimchi, A. Vishwanath, and J. G. Analytis, Nature (London) 535, 266 (2016).

[17] C. M. Wang, H.-P. Sun, H.-Z. Lu, and X. C. Xie, Phys. Rev. Lett. 119, 136806 (2017).

[18] C. Zhang, A. Narayan, S. Lu, J. Zhang, H. Zhang, Z. Ni, X. Yuan, Y. Liu, J.-H. Park, E. Zhang, W. Wang, S. Liu, L. Cheng,
L. Pi, Z. Sheng, S. Sanvito, and F. Xiu, Nat. Commun. 8, 1272 (2017).

[19] C. Zhang, Y. Zhang, X. Yuan, S. Lu, J. Zhang, A. Narayan, Y. Liu, H. Zhang, Z. Ni, R. Liu, E. S. Choi, A. Suslov, S. Sanvito, L. Pi, H.-Z. Lu, A. C. Potter, and F. Xiu, Nature (London) 565, 331 (2018).

[20] E. Witten, Commun. Math. Phys. 121, 351 (1989).

[21] T. Bzdušek, Q. Wu, A. Rüegg, M. Sigrist, and A. A. Soluyanov, Nature (London) 538, 75 (2016).

[22] W. Chen, H.-Z. Lu, and J.-M. Hou, Phys. Rev. B 96, 041102(R) (2017).

[23] Z. Yan, R. Bi, H. Shen, L. Lu, S.-C. Zhang, and Z. Wang, Phys. Rev. B 96, 041103(R) (2017).

[24] M. Ezawa, Phys. Rev. B 96, 041202(R) (2017).

[25] G. Chang, S.-Y. Xu, X. Zhou, S.-M. Huang, B. Singh, B. Wang, I. Belopolski, J. Yin, S. Zhang, A. Bansil, H. Lin, and M. Z. Hasan, Phys. Rev. Lett. 119, 156401 (2017).

[26] R. Bi, Z. Yan, L. Lu, and Z. Wang, Phys. Rev. B 96, 201305(R) (2017).

[27] In comparison, the nodal link and nodal knot metals rest their topological distinctions on the nodal line topology in threedimensional momentum space. They remain gapless in the presence of a magnetic field and disperse in the momentum space along the magnetic field. Therefore, their nodal links and knots do not translate to their cyclotron orbits in three spatial dimensions.

[28] See Supplemental Material at http://link.aps.org/supplemental/ 10.1103/PhysRevResearch.1.022005 for the illustrative plot and model construction guidelines in Supplemental Materials.

[29] The $C_{3}$ rotation symmetry is for simplicity and not a necessary ingredient of the cyclotron orbit knotting geometry.

[30] D. Xiao, M.-C. Chang, and Q. Niu, Rev. Mod. Phys. 82, 1959 (2010).

[31] G. Chang, S.-Y. Xu, B. J. Wieder, D. S. Sanchez, S.-M. Huang, I. Belopolski, T.-R. Chang, S. Zhang, A. Bansil, H. Lin, and M. Z. Hasan, Phys. Rev. Lett. 119, 206401 (2017). 